\title{
Robotic revision surgery for type IV choledochal cyst
}

\author{
Raja KALAYARASAN*
}

Department of Surgical Gastroenterology, JIPMER, Puducherry, India

Introduction: Complete excision of the cyst with Roux-en-Y hepaticojejunostomy (RYHJ) is the standard treatment for a choledochal cyst (CDC). Incomplete cyst excision and anastomotic stricture are the common causes of recurrent symptoms. The use of the robotic platform for re-operation after open surgical treatment of the CDC has not been previously reported. The technique of robotic revision surgery using the daVinci ${ }^{\circledR} \mathrm{XiTM}$ robotic surgical system is described in this report.

Methods: A 24-year-old female with a history of open CDC excision and choledochoduodenostomy at three months of age, presented with intermittent fever and abdominal pain for ten years duration. Preoperative evaluation with upper gastrointestinal endoscopy and magnetic resonance imaging revealed choledochoduodenostomy stricture with cystic dilatation of biliary radicals predominantly in the left lobe liver suggestive of type IVa choledochal cyst. The patient underwent robotic dismantling of strictured choledochoduodenostomy, left hepatectomy and Roux-en-Y right hepaticojejunostomy. The procedure was performed with four $8 \mathrm{~mm}$ robotic trocars placed at the umbilicus level and one infraumbilical assistant trocar. Key steps are intra-abdominal adhesiolysis, dismantling of the choledochoduodenostomy, inflow control to the left hemiliver, parenchymal transection to the left of the middle hepatic vein, division of left hepatic duct, intraparenchymal transection of the left hepatic vein and Roux-en-Y right hepaticojejunostomy.

Results: The operative time, blood loss, and postoperative hospital stay were 410 minutes, $200 \mathrm{~mL}$, and four days, respectively. At seven months follow-up, the patient is asymptomatic with normal liver function test.

Conclusions: Robotic revision surgery for CDC is safe and the feasibility reported in the present patient needs to be documented in a large case series. 